\title{
Bipolar doping and thermoelectric properties of Zintl arsenide $\mathrm{Eu}_{5} \ln _{2} \mathrm{As}_{6}$
}

\begin{abstract}
Naoki Tomitaka, ${ }^{\text {a }}$ Yosuke Goto, ${ }^{* a}$ Kota Morino, ${ }^{a}$ Kazuhisa Hoshi, ${ }^{a}$ Yuki Nakahira, ${ }^{a}$ Hiroaki Ito, ${ }^{\text {b }}$ Akira Miura, ${ }^{b}$ Hidetomo Usui, ${ }^{* c}$ Yoshikazu Mizuguchi ${ }^{a}$

Zintl compounds exhibit promising thermoelectric properties because of the feasibility of the chemical tuning of their electrical and thermal transport. While most Zintl pnictides are known to show $p$-type polarity, recent developments in highperformance $\mathrm{n}$-type $\mathrm{Mg}_{3} \mathrm{Sb}_{2}$-based thermoelectric materials have encouraged further identification of $\mathrm{n}$-type Zintl pnictides. In this study, we demonstrate the bipolar dopability of the Zintl arsenide $\mathrm{Eu}_{5} \mathrm{In}_{2} \mathrm{As}_{6}$. The electrical resistivity at $300 \mathrm{~K}$ with $\mathrm{n}$ type polarity was decreased to $7.6 \times 10^{-1} \Omega \mathrm{cm}$ using La as an electron dopant. In contrast to the relatively high resistivity of $\mathrm{n}$-type $\mathrm{Eu}_{5} \mathrm{In}_{2} \mathrm{As}_{6}$, the p-type resistivity at $300 \mathrm{~K}$ was decreased to $5.9 \times 10^{-3} \Omega \mathrm{cm}$ with a carrier concentration of $2.8 \times 10^{20}$ $/ \mathrm{cm}^{3}$ using $\mathrm{Zn}$ as a hole dopant. This doping asymmetry is discussed in terms of the weighted mobility of electrons and holes. Furthermore, a very low lattice thermal conductivity of $0.7 \mathrm{~W} / \mathrm{mK}$ was observed at $773 \mathrm{~K}$, which is comparable to that of the

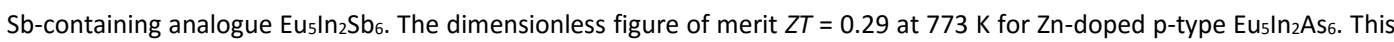
study shows that bipolar dopable $\mathrm{Eu}_{5} \ln _{2} \mathrm{As}_{6}$ can be a platform to facilitate a better understanding of the doping asymmetry in Zintl pnictides.
\end{abstract}

\section{Introduction}

Controlling the charge carrier polarity and concentration is essential for the development of novel semiconductor devices. For example, the thermoelectric module enables interconversion between temperature difference and electricity, and is a promising component for energy harvesting from waste heat. ${ }^{1-4}$ The conventional thermoelectric module requires the integration of $p$ type and $n$-type semiconductors. The performance of thermoelectric materials is characterised by a dimensionless figure of merit, $Z T=S^{2} T \rho^{-1} \kappa^{-1}$, where $T$ is the absolute temperature, $S$ is the Seebeck coefficient, $\rho$ is the electrical resistivity, and $\kappa$ is the thermal conductivity. Therefore, both $\mathrm{p}$-type and n-type semiconductors that show high $Z T$ values are required to construct efficient thermoelectric modules.

Various thermoelectric materials have been investigated over the past several decades. Among them, Zintl phase pnictides, which consist of covalently bonded anionic frameworks and cations, have been studied extensively because of their fascinating compositional/structural varieties and promising thermoelectric properties. ${ }^{5-7}$ For example, $A B_{2} X_{2}, A_{14} B X_{11}, A_{3} B X_{3}, A_{5} B_{2} X_{6}, A_{9} B_{4+\delta} X_{9}$, and $A_{2} B_{2}$-where $A$ denotes an alkali/alkaline-earth/rare-earth metal, $B$ is a (post-) transition metal, and $X$ is a pnictogen-have been demonstrated as promising thermoelectric materials, ${ }^{8-15}$

\footnotetext{
a. Department of Physics, Tokyo Metropolitan University, 1-1 Minami-osawa, Hachioji, Tokyo 192-0397, Japan. E-mail:y_goto@tmu.ac.jp

b. Faculty of Engineering, Hokkaido University, Kita 13, Nishi 8 Sapporo 060-8628, Japan

c. Department of Physics and Materials Science, Shimane University, Matsue, 690 8504, Japan.E-mail: hidetomo.usui@riko.shimane-u.ac.jp
}

owing to the complex crystal structure that can lead to intrinsically lattice thermal conductivity, while the presence of covalent bonding maintains moderately high carrier mobility. Such characteristics offer promise for efficient thermoelectrics as well as other state-ofthe arts thermoelectric materials, including GeTe, ${ }^{16,17} \mathrm{SnSe}{ }^{18}$ SnTe, ${ }^{19}$ flexible materials, ${ }^{20}$ oxides, ${ }^{21}$ etc.

While most Zintl pnictides are known to show p-type polarity, the recent development of high-performance $\mathrm{n}$-type $\mathrm{Mg}_{3} \mathrm{Sb}_{2}$-based thermoelectric materials, which exhibit $Z T=1.51$ at $716 \mathrm{~K},{ }^{13,14}$ has encouraged further identification of $n$-type Zintl pnictides. However, fabricating n-type Zintl pnictides is not an easy task because most of the n-type doping is compensated by the so-called killer defects, such as cation vacancy. ${ }^{22}$ In addition to $\mathrm{Mg}_{3} \mathrm{Sb}_{2}$-based materials, $\mathrm{ABX}_{4},{ }^{23,24} \mathrm{Eu}_{5-x} \mathrm{La}_{x} \mathrm{In}_{2.2} \mathrm{Sb}_{6},{ }^{25} \mathrm{Eu}_{3} \ln \mathrm{As}_{3},{ }^{26}$ and $\mathrm{Ba}_{3} \mathrm{Cd}_{2} \mathrm{P}_{4}{ }^{27}$ have been reported as n-type Zintl pnictides so far.

The crystal structure of Zintl arsenide $\mathrm{Eu}_{5} \mathrm{In}_{2} \mathrm{As}_{6}$ belongs to the orthorhombic space group Pbam, as shown in Figure 1.28,29 Each In atom is four-fold coordinated by As atoms to form InAs $s_{4}$ tetrahedra, which are corner-shared along the $c$ axis. The chains of InAs 4 tetrahedra are further bridged by As-As bonds. The Eu atoms between the chains provide electrons to the covalently bonded anionic framework, yielding an overall charge balance. Mössbauer spectroscopy suggests the valence state as $\mathrm{Eu}^{2+}{ }_{5} \mathrm{In}^{3+}{ }_{2} \mathrm{As}^{3-}{ }_{4} \mathrm{As}^{2-}{ }_{2}$, while Zintl-concept describes that as $\mathrm{Eu}^{2+}{ }_{5} \mathrm{In}^{-}{ }_{2} \mathrm{As}^{-}{ }_{4} \mathrm{As}^{2-}{ }_{2}$. This material undergoes two-step magnetic transitions at $5 \mathrm{~K}$ and $16 \mathrm{~K}$, likely because of $\mathrm{Eu}^{2+}$ ions that include three distinct crystallographic sites. ${ }^{29}$

In this study, we demonstrate that Zintl arsenide $\mathrm{Eu}_{5} \mathrm{In}_{2} \mathrm{As}_{6}$ is a bipolar dopable material. La and $\mathrm{Zn}$ were used as the $\mathrm{n}$-type and $\mathrm{p}$ type dopants, respectively, because these elements have been 


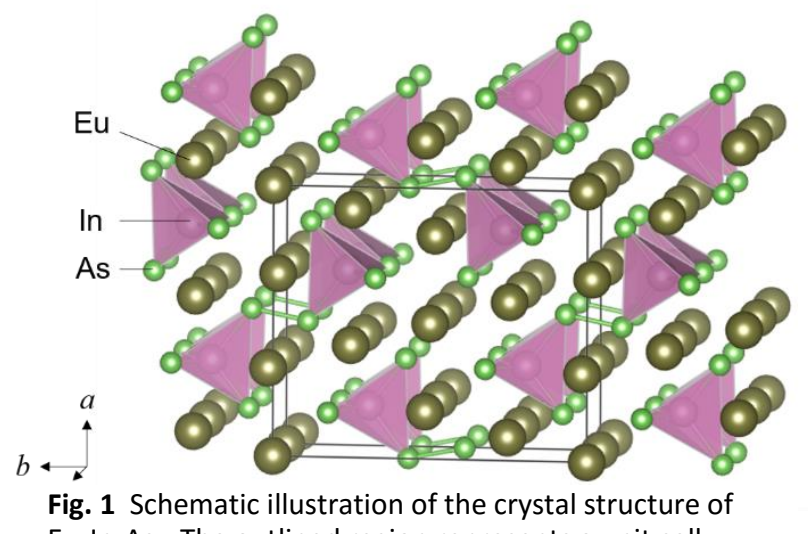

Fig. 1 Schematic illustration of the crystal structure of
$\mathrm{Eu}_{5} \operatorname{In}_{2} \mathrm{As}_{6}$. The outlined region represents a unit cell.

investigated as effective dopants in the sister compound $E u_{5} I_{2} S_{6} b_{6} .25,30$ While $n$-type $E u_{5} \ln _{2} A_{s_{6}}$ shows relatively high electrical resistivity of $\sim 10^{-1} \Omega \mathrm{cm}$, p-type samples exhibit resistivity as low as $\sim 10^{-3} \Omega \mathrm{cm}$. This doping asymmetry is discussed in terms of the weighted mobility of electrons and holes. Furthermore, a very low lattice thermal conductivity of $0.7 \mathrm{~W} / \mathrm{mK}$ was observed at $773 \mathrm{~K}$. First-principles calculations were performed to investigate the electronic structure and transport properties.

\section{Method}

\section{Sample preparation}

Polycrystalline Eu $\mathrm{In}_{2} \mathrm{~A} \mathrm{~s}_{6}$ was prepared by reacting EuAs, InAs, and In (99.99\%) powder, which is similar to the method employed in our previous studies. ${ }^{31,32}$ EuAs was prepared by reacting Eu (99.9\%) with As $(99.9999 \%)$ in a $1: 1$ stoichiometric ratio at $850^{\circ} \mathrm{C}$ for $10 \mathrm{~h}$ in a carbonised silica tube. We scraped the surface of the Eu ingot mechanically by filing it before use. We then cut or crushed the Eu ingot and As grains into small pieces to ensure the feasibility of the reaction by increasing the surface area. The silica tube was carbonised via the evaporation of acetone. InAs was prepared by reacting In with As in a $1: 1$ stoichiometric ratio at $950{ }^{\circ} \mathrm{C}$ for $10 \mathrm{~h}$ in a sealed silica tube. We then prepared a stoichiometric mixture of EuAs, InAs, and In in a 1:1:1 ratio, which was then pelletised, placed in a carbon crucible, and heated at $800{ }^{\circ} \mathrm{C}$ for $30 \mathrm{~h}$ in a sealed silica tube. We also prepared $E u_{5-x} \operatorname{La}_{x} \mid \mathrm{n}_{2} \mathrm{As}_{6}(x=0.05,0.10)$ and $E u_{5} \ln _{2-y} Z_{y} A s_{6}(y=0.05,0.10$, and 0.15$)$. LaAs was added to the precursor for the synthesis of $\mathrm{Eu}_{5-x} \mathrm{La}_{x} \mathrm{In}_{2} \mathrm{As}_{6}$. LaAs was prepared by reacting La (99.9\%) with As in a $1: 1$ stoichiometric ratio at $700{ }^{\circ} \mathrm{C}$ for $20 \mathrm{~h}$ in a silica tube. For the synthesis of $\mathrm{Eu}_{5} \mathrm{In}_{2-y} \mathrm{Zn}_{y} \mathrm{As}_{6}, \mathrm{Zn}(99.9 \%)$ was added to the precursor.

We compressed the obtained samples by hot-pressing them in a graphite die at $50 \mathrm{MPa}$ and $650-700{ }^{\circ} \mathrm{C}$ for $30 \mathrm{~min}$. We calculated the geometric density of each sample to be $>95 \%$ of the corresponding unit cell density. The obtained samples were observed to endure at least several weeks of exposure to air without visible signs of oxidation/hydrolysis.

\section{Sample characterisation}

We examined particle size and morphology of the obtained samples using scanning electron microscopy (SEM; Hitachi TM3030). We investigated the chemical compositions using energy-dispersive $\mathrm{X}$ - ray spectrometry (EDX; Oxford SwiftED3000). We measured the Xray diffraction (XRD) patterns in the laboratory with CuK $\alpha$ radiation using a Rigaku MiniFlex 600 equipped with a D/teX Ultra detector. In addition, we measured the SXRD patterns at BL02B2 of SPring-8 under proposal number 2021A1573. The diffraction data were obtained using a high-resolution one-dimensional semiconductor detector (MYTHEN). ${ }^{33}$ The wavelength of the radiation beam was determined to be 0.495810 (1) $\AA$ using $\mathrm{a} \mathrm{CeO}_{2}$ standard. The crystal structure parameters were refined by the Rietveld method using JANA $2020^{34}$ using the diffraction intensity data in the reciprocal space of $\sin \theta / \lambda<0.52 / \AA$ (lattice spacing $d>0.96 \AA$ ). The crystal structure was visualised using VESTA. ${ }^{35}$

\section{Thermoelectric transport properties}

We measured the Hall coefficient $R_{\mathrm{H}}$ using the five-probe method with a physical property measurement system (Quantum Design). We calculated the Hall carrier concentration as $n_{\mathrm{H}}=1 / R_{\mathrm{H}} e$, where $e$ is the charge of an electron. We measured the electrical resistivity and Seebeck coefficient using a four-probe method and a quasisteady-state method, respectively (ZEM-3, Advance Riko). The thermal diffusivity was measured using a laser flash method (TC1200RH, Advance Riko). We calculated the thermal conductivity using the relationship $k=D C_{p} d$, where $D, C_{p}$, and $d$ are the thermal diffusivity, specific heat, and sample density, respectively. We estimated $C_{p}$ using the Dulong-Petit law, $C_{p}=3 n_{a} R$, where $n_{a}$ is the number of atoms per formula unit, and $R$ is the gas constant.

The thermal hysteresis of the samples used in this study is almost negligible, at least up to $773 \mathrm{~K}$.

\section{First-principles calculation}

We performed first-principles calculations within density functional theory (DFT) using VASP 5.4.4 ${ }^{36,37}$ to obtain the electronic structure of $E u_{5} I_{2} \mathrm{As}_{6}$ by adopting the experimentally determined structural parameters. We calculated the electronic density, including spinorbit coupling, self-consistently with the modified Becke-Johnson potential ${ }^{38}$ using a $3 \times 3 \times 8 k$-mesh. The cut-off energy for the plane-wave basis set was fixed at $350 \mathrm{eV}$. For Eu, we used the potentials obtained with open-core treatment for the $f$-electrons. We calculated the Seebeck coefficient within the Boltzmann transport theory, as implemented in the BoltzTraP code, ${ }^{39}$ using an $11 \times 10 \times 29 k$-mesh. We assumed the rigid-band approximation and a constant relaxation time to calculate the Seebeck coefficient tensors. For a comparison with the experimental results for polycrystalline samples, we obtained the final results for the Seebeck coefficients by averaging the $x x, y y$, and $z z$ components of the Seebeck coefficient tensor.

\section{Results}

\section{Sample characterisation}

The chemical compositions determined using EDX are summarised in Table 1. The analysed composition is reasonably consistent with the nominal composition of the starting materials. SEM images show that the obtained samples are $\mu \mathrm{m}$-sized particles, as shown in Figure S1. 
Table 1 Chemical compositions obtained using EDX and the lattice parameters of $E u_{5-x} L_{x} \mid n_{2} A s_{6}$ and $E u_{5} \ln _{2-y} Z n_{y} A s_{6}$.

\begin{tabular}{cccccc}
\hline Nominal composition & \multicolumn{2}{c}{ Analysed composition } & \multicolumn{4}{c}{ Lattice parameters } \\
\hline $\mathrm{Eu}_{5-x} \mathrm{La}_{x} \mathrm{I}_{2} \mathrm{As}_{6}$ & Eu:La:In:As & $a(\AA)$ & $b(\AA)$ & $c(\AA)$ & $V\left(\AA^{3}\right)$ \\
$x=0$ & $4.7(2): 0: 2.04(6): 6.3(3)$ & $11.89005(4)$ & $13.79079(4)$ & $4.350601(13)$ & $713.38(4)$ \\
0.05 & $4.69(5): 0.01(1): 2.03(5): 6.27(7)$ & $11.88898(7)$ & $13.79293(8)$ & $4.35021(2)$ & $713.364(7)$ \\
0.10 & $4.57(7): 0.03(2): 2.02(4): 6.4(1)$ & $11.88856(15)$ & $13.79437(17)$ & $4.35080(5)$ & $713.51(2)$ \\
$\mathrm{Eu}_{5} \mathrm{In}_{2-y} \mathrm{Zn}_{y} \mathrm{As} 6$ & $\mathrm{Eu}: \operatorname{In}: \mathrm{Zn}: \mathrm{As}$ & & & & \\
$y=0.05$ & $4.6(1): 1.99(4): 0.01(1): 6.4(1)$ & $11.89106(6)$ & $13.78907(8)$ & $4.34756(2)$ & $712.855(6)$ \\
0.10 & $4.65(8): 1.95(8): 0.03(2) 6.4(1)$ & $11.89062(6)$ & $13.78546(7)$ & $4.34384(2)$ & $712.032(6)$ \\
0.15 & $4.6(1): 2.0(1) ; 0.04(3) 6.4(2)$ & $11.89168(19)^{\mathrm{a}}$ & $13.7879(2)^{\mathrm{a}}$ & $4.34427(7)^{\mathrm{a}}$ & $712.29(2)^{\mathrm{a}}$ \\
\hline
\end{tabular}

aLattice parameters of $y=0.15$ was obtained using laboratory XRD measurements.

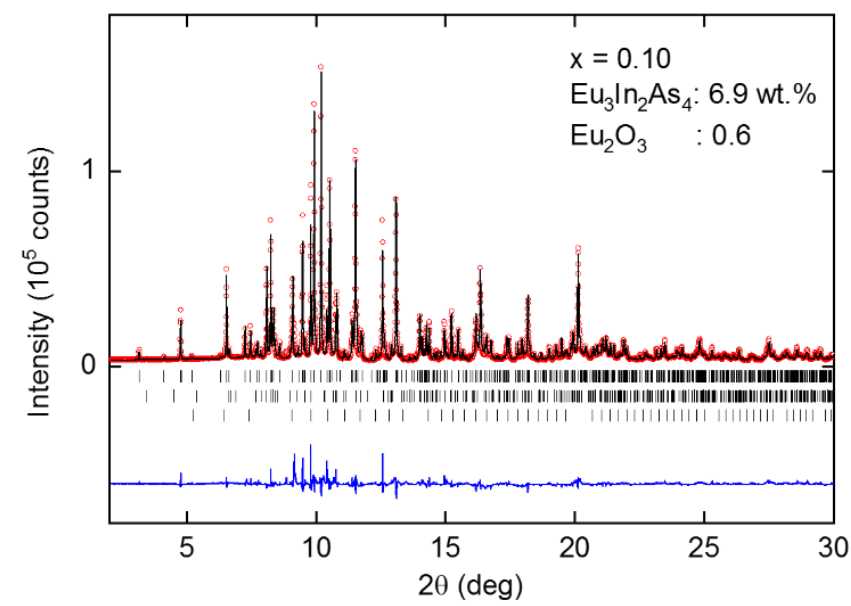

Fig. 2 Observed SXRD pattern of Eu $u_{4.90} \operatorname{La}_{0.10} \ln _{2} A s_{6}$ and the Rietveld refinement results. The circles (red) and solid lines (black) represent the observed and calculated patterns, respectively. The difference between the observed and calculated patterns is shown at the bottom (blue). The vertical marks indicate the Bragg reflection positions for $\mathrm{Eu}_{4.90} \mathrm{La}_{0.10} \mathrm{In}_{2} \mathrm{As}_{6}, \mathrm{Eu}_{3} \mathrm{In}_{2} \mathrm{As}_{4}$, and $\mathrm{Eu}_{2} \mathrm{O}_{3}$, respectively, from top to bottom. The reliability factors are $R_{\mathrm{wp}}=8.35 \%, R_{\mathrm{p}}=$ $6.78 \%$, and $\mathrm{GOF}=9.70$. Analogous data for other samples are shown in Figure S2 in ESI.

Figure 2 shows the SXRD pattern and Rietveld refinement results for $\mathrm{Eu}_{4.90} \mathrm{La}_{0.10} \mathrm{In}_{2} \mathrm{As} \mathrm{s}_{6}$ as a representative sample. Most of the diffraction peaks can be assigned to the space group Pbam, indicating that the $\mathrm{Eu}_{5} \mathrm{In}_{2} \mathrm{As}_{6}$-type structure is the primary phase. We estimated the amounts of impurity phases using Rietveld refinement: $\mathrm{Eu}_{3} \mathrm{In}_{2} \mathrm{As}_{4}(6.9 \mathrm{wt} \%)$ and $\mathrm{Eu}_{2} \mathrm{O}_{3}(0.6 \mathrm{wt} \%)$. Note that oxide impurities $\left(\mathrm{Eu}_{2} \mathrm{O}_{3}\right)$ are likely derived from the raw Eu ingots. Furthermore, it may also be possible that carbonised layer of the silica tube acts as an oxygen source because of uncompletedburned organic residues. The lattice parameters of $E u_{5-x} L_{x} I_{2}{ }_{2} A s_{6}$ and $E u_{5} I_{2-y} Z n_{y} A s_{6}$ are listed in Table 1. For undoped Eu $\ln _{2} A s_{6}$, the lattice parameters are consistent with previously reported results. ${ }^{29,30}$ The lattice volume tends to decrease with increasing $\mathrm{Zn}$ content, which is consistent with the smaller ionic radius of $\mathrm{Zn}^{2+}$ than that of $\ln ^{3+}$ (Shannon's four-coordinated ionic radius, $r\left(\mathrm{Zn}^{2+}\right)=$ $0.60 \AA$ and $\left.r\left(\mathrm{In}^{3+}\right)=0.62 \AA\right) .{ }^{40}$ Although the lattice volume is slightly different in La-doped samples, systematic change with La content is not observed, despite a smaller ionic radius of $\mathrm{La}^{3+}$ than that of $\mathrm{Eu}^{2+}$ $\left(r\left(\mathrm{La}^{3+}\right)=1.03 \AA\right.$ and $\left.r\left(\mathrm{Eu}^{2+}\right)=1.17 \AA\right) .{ }^{40}$ This result suggests that La doping may be compensated by some defects.
Rietveld refinement of site occupancy gives $\ln : Z n=0.97: 0.03$ for Eu $\mathrm{In}_{1.9} \mathrm{Zn}_{0.1} \mathrm{As}_{6}$, indicating that $\mathrm{Zn}$ dopants indeed occupy the In site. Although we performed site occupancy analysis for La-doped samples, it is difficult to obtain reasonable results, likely because of similar electron numbers of Eu and La. The Rietveld refinement results for the other samples are shown in Figure S2 and Table S1 in ESI. Hereafter, we denote the samples using the nominal composition of the starting materials for simplicity.

\section{Charge-carrier transport}

Figure 3 shows the electrical resistivity and Seebeck coefficient at $300 \mathrm{~K}$. The electrical resistivity of undoped $\mathrm{Eu}_{5} \mathrm{In}_{2} \mathrm{As}_{6}$ is $7.2 \Omega \mathrm{cm}$, which is decreased by La or $\mathrm{Zn}$ doping; the corresponding values for $\mathrm{Eu}_{4.90} \mathrm{La}_{0.10} \mathrm{In}_{2} \mathrm{As}_{6}$ and $\mathrm{Eu}_{5} \mathrm{In}_{1.85} \mathrm{Zn}_{0.15} \mathrm{As}_{6}$ are $7.6 \times 10^{-1} \Omega \mathrm{cm}$ and $5.9 \times$ $10^{-3} \Omega \mathrm{cm}$, respectively. Thus, $\mathrm{Zn}$ is a more effective dopant for inducing carriers in $\mathrm{Eu}_{5} \mathrm{In}_{2} \mathrm{As}_{6}$. The Seebeck coefficient of the undoped sample is $-32 \mu \mathrm{V} / \mathrm{K}$, that is, undoped $\mathrm{Eu}_{5} \ln _{2} \mathrm{As}_{6}$ already exhibits n-type polarity, because the sign of the Seebeck coefficient is a measure of the carrier polarity. This is in contrast to the $p$-type polarity of most Zintl pnictides. Despite the high resistivity of the undoped sample $(7.2 \Omega \mathrm{cm})$, the absolute value of the Seebeck coefficient was not significantly high. This is likely because of the multicarrier nature of the undoped sample, ${ }^{41}$ namely, the coexistence of electrons and holes, which is also suggested by the temperature dependence of the Seebeck coefficient, as shown later. The Seebeck coefficient of the La/Zn-doped samples shows a negative/positive sign. The results indicate that $\mathrm{La} / \mathrm{Zn}$ acts as an electron/hole dopant in $\mathrm{Eu}_{5} \mathrm{In}_{2} \mathrm{As}_{6}$, as expected from the simple ionic valence counting ( $\mathrm{La}^{3+}$-doping into $\mathrm{Eu}^{2+}$, and $\mathrm{Zn}^{2+}$-doping into $\left(\mathrm{n}^{3+}\right)$. For the $\mathrm{Zn}$-doped sample, the Seebeck coefficient decreased to $96 \mu \mathrm{V} / \mathrm{K}$, which is consistent with the decrease in electrical resistivity.

The Hall coefficient of undoped $\mathrm{Eu}_{5} \mathrm{In}_{2} \mathrm{As}_{6}$ was measured to be $-35.4 \mathrm{~cm}^{3} / \mathrm{C}$. The negative sign of the Hall coefficient confirms the n-type polarity of $\mathrm{Eu}_{5} \mathrm{In}_{2} \mathrm{As}_{6}$, which is consistent with the Seebeck coefficient. The Hall carrier concentration of Eu $\mathrm{In}_{2} \mathrm{As}_{6}$ was determined to be $1.8 \times 10^{17} / \mathrm{cm}^{3}$, and it increased with increasing $\mathrm{Zn}$ content. As shown in Figure 4 , an increase in carrier concentration with $\mathrm{Zn}$ content is reasonably consistent with the expected value, assuming that each $Z n$ generates one free hole, although the experimental carrier concentration is slightly lower than the expected value. Unlike for the $\mathrm{Zn}$-doped samples, it is 
(a)

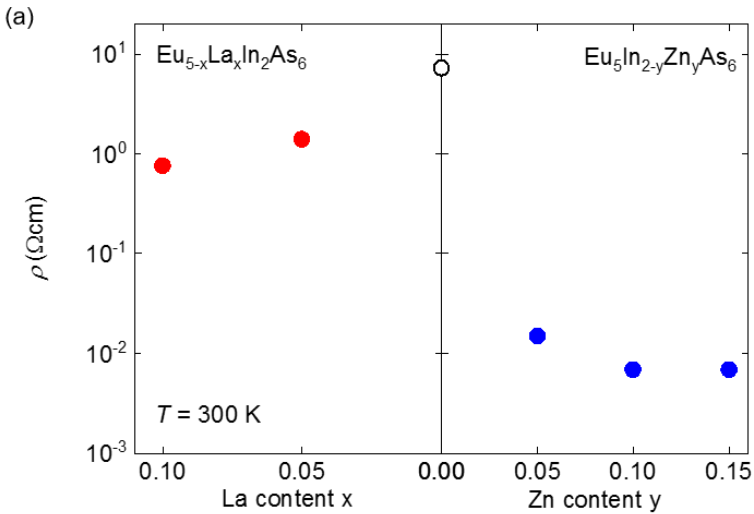

(b)

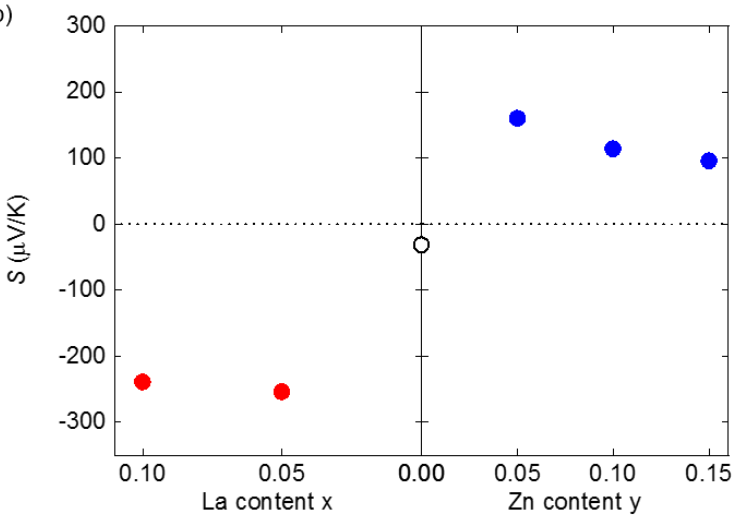

Fig. 3 (a) Electrical resistivity $\rho$ and (b) Seebeck coefficient $S$ of $E u_{5-x} L_{x} I_{2} A s_{6}$ and $E u_{5} I_{2-y} Z n_{y} A s_{6}$ at $300 \mathrm{~K}$.

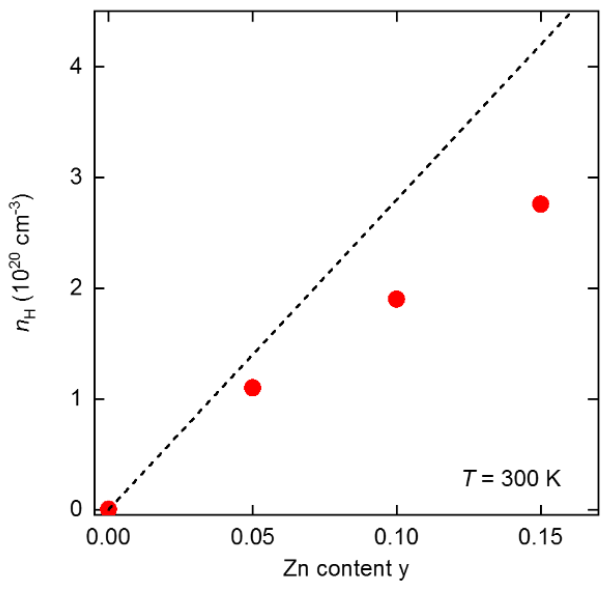

Fig. 4 Hall carrier concentration of Eu $\operatorname{In}_{2-y} Z n_{y} A s_{6}$ at $300 \mathrm{~K}$. The dashed line represents the expected value, assuming each $\mathrm{Zn}$ provides one free hole.

difficult to obtain reliable Hall coefficients of the La-doped samples, probably because of their low electron mobility. To discuss the mobility of these samples, we calculated the weighted mobility $\mu_{\mathrm{w}}$, that is, the carrier mobility weighted by the density of electronic states, using their electrical resistivity and Seebeck coefficient. ${ }^{42}$ It has been reported that the weighted mobility provides a reasonable assessment of carrier mobility even if the materials have a low mobility. As listed in Table 2, the magnitude of the weighted mobility of the $\mathrm{Zn}$-doped samples was $\sim 10 \mathrm{~cm}^{2} / \mathrm{Vs}$, whereas that of the La-doped samples was $\sim 10^{-1} \mathrm{~cm}^{2} / V$ s. These results confirm the difficulty in measuring the Hall coefficient of La-doped samples due to low electron mobility. We estimated Hall coefficient of La-doped samples at at $0.5 \mathrm{~cm}^{3} / \mathrm{C}\left(E_{4.95} L_{0.05} I_{2} \mathrm{As}_{6}\right)$ and $1.3 \mathrm{~cm}^{3} / \mathrm{C}$ $\left(E u_{4.90} L_{0.10} I_{2} A_{5}\right)$, using measured electrical resistivity and calculated weighted mobility. However, these values might be overestimated because weighted mobility tends to be higher than Hall mobility (Table 2).

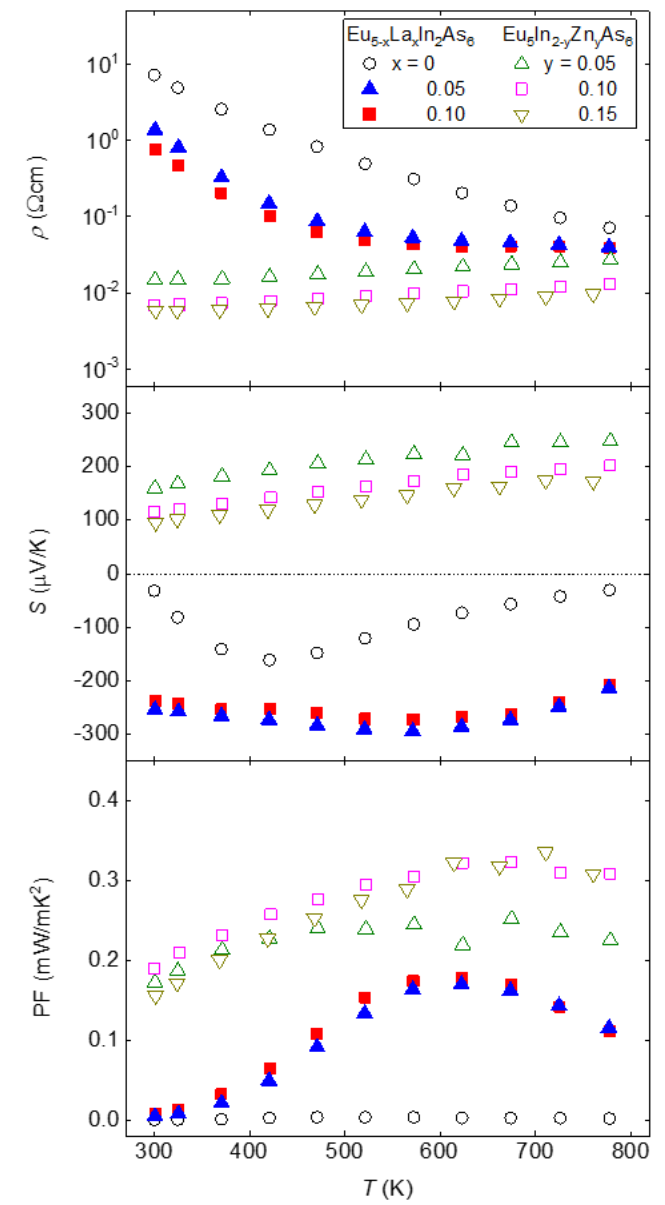

Fig. 5 Electrical resistivity $\rho$, Seebeck coefficient $S$, and electrical power factor PF as functions of temperature $T$ for $\mathrm{Eu}_{5-x} \mathrm{La}_{x} \mathrm{In}_{2} \mathrm{As}_{6}$ and $E u_{5} \mathrm{In}_{2-y} \mathrm{Zn}_{y} \mathrm{As}_{6}$. 
(a)

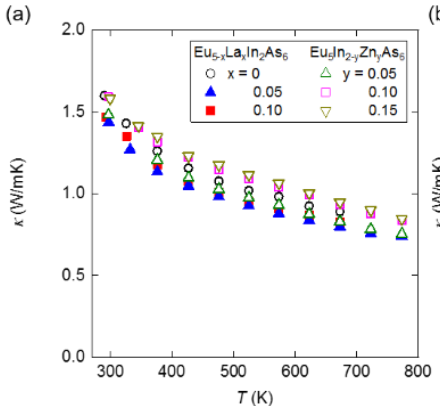

(b)

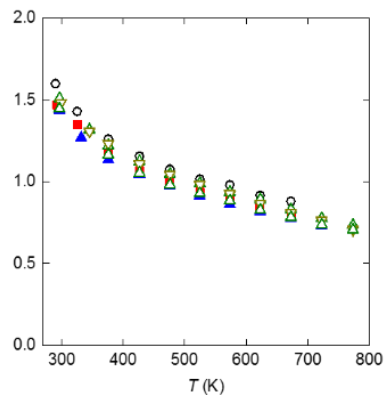

Fig. 6 Temperature $(T)$ dependence of the (a) total thermal conductivity $\kappa$ and (b) lattice thermal conductivity $\kappa$ of $\mathrm{Eu}_{5-x} \mathrm{La}_{x} \mathrm{In}_{2} \mathrm{As}_{6}$ and $\mathrm{Eu}_{5} \mathrm{In}_{2-y} \mathrm{Zn}_{y} \mathrm{As}_{6}$.

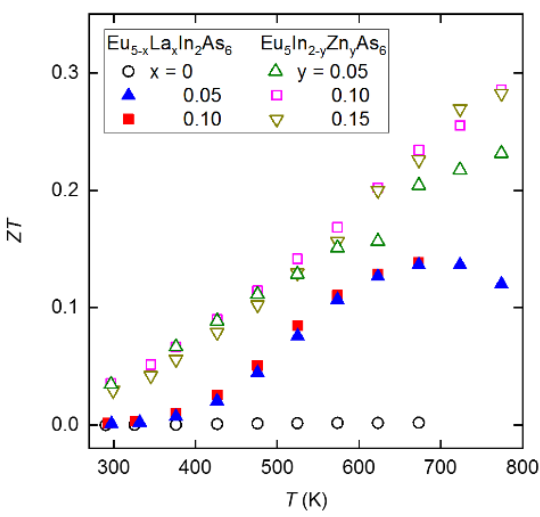

Fig. 7 Dimensionless figure of merit $Z T$ as a function of temperature $T$ for $E u_{5-x} L_{x} I_{n} A s_{6}$ and $E u_{5} \ln _{2-y} Z_{y} A s_{6}$.

Figure 5 shows the temperature dependence of the electrical resistivity, Seebeck coefficient, and power factor $\left(P F=\rho^{-1} S^{2}\right)$ of $E u_{5-x} L_{x} I_{2} A s_{6}$ and $E u_{5} \ln _{2-y} Z n_{y} A s_{6}$. The undoped sample shows a semiconducting behaviour with high resistivity, and the activation energy is evaluated to be $0.29 \mathrm{eV}$ using the Arrhenius plot ( $\ln \rho$ vs. $T^{-1}$ ) in the high-temperature region, as shown in Figure S3. Although the electrical resistivity of the La-doped samples is decreased, their semiconducting behaviour is still maintained. In contrast, degenerate conduction (positive temperature coefficient) is observed for the $\mathrm{Zn}$-doped samples.

The Seebeck coefficient of the undoped sample shows a maximum at approximately $420 \mathrm{~K}$, and the absolute value of the Seebeck coefficient decreases with increasing temperature above this temperature, indicating the multicarrier nature of undoped $\mathrm{Eu}_{5} \mathrm{In}_{2} \mathrm{As}_{6}$. This is consistent with its low electron mobility, as listed in Table 2. The Seebeck coefficient of the La-doped samples still exhibits a maximum at approximately $570 \mathrm{~K}$. In contrast, the Seebeck coefficient of the $\mathrm{Zn}$-doped samples increases with increasing temperature without a maximum.

The calculated power factor of the La-doped samples is less than $0.2 \mathrm{~mW} / \mathrm{mK}^{2}$ because of their high electrical resistivity. The $\mathrm{Zn}$ doped samples exhibit a power factor of approximately 0.3 $\mathrm{mW} / \mathrm{mK}^{2}$ over a broad temperature range $(500-800 \mathrm{~K})$, which is comparable to that of the Sb-containing analogue $\mathrm{Eu}_{5} \mathrm{In}_{2} \mathrm{Sb}_{6} \cdot{ }^{30}$

\section{Thermal transport and thermoelectric figure-of-merit}

Table 2 Hall carrier concentration $n_{\mathrm{H}}$, Hall carrier mobility $\mu_{\mathrm{H}}$ and weighted mobility $\mu_{w}$ for $E u_{5-x} L_{x} I n_{2} A s_{6}$ and $E u_{5} I n_{2-y} Z n_{y} A s_{6}$ at $300 \mathrm{~K}$. A reliable Hall coefficient was not obtained for $\mathrm{Eu}_{5-x} \operatorname{La}_{x} \operatorname{In}_{2} \mathrm{As}_{6}(x=0.05$ and 0.10$)$.

\begin{tabular}{llll}
\hline Sample & $n_{\mathrm{H}}\left(\mathrm{cm}^{-3}\right)$ & $\mu_{\mathrm{H}}\left(\mathrm{cm}^{2} / \mathrm{Vs}\right)$ & $\mu_{\mathrm{W}}\left(\mathrm{cm}^{2} / \mathrm{Vs}\right)$ \\
\hline $\mathrm{Eu}_{5-x} \mathrm{La}_{x} \mathrm{In}_{2} \mathrm{As}_{6}$ & & & \\
$x=0$ & $1.8 \times 10^{17}$ & 4.9 & $5.3 \times 10^{-3}$ \\
0.05 & -- & -- & $6.1 \times 10^{-1}$ \\
0.10 & -- & -- & $9.4 \times 10^{-1}$ \\
$\mathrm{Eu}_{5} \mathrm{In}_{2-y} \mathrm{Zn}_{y} \mathrm{As}_{6}$ & & & \\
$y=0.05$ & $1.1 \times 10^{20}$ & 3.7 & 19.2 \\
0.10 & $1.9 \times 10^{20}$ & 4.7 & 23.5 \\
0.15 & $2.8 \times 10^{20}$ & 3.8 & 21.6 \\
\hline
\end{tabular}

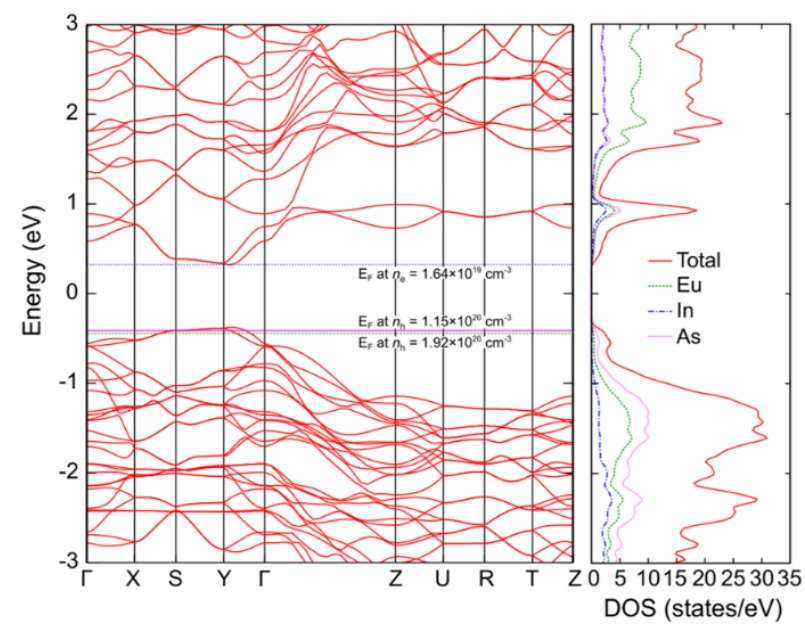

Fig. 8 Band structure and density of states (DOS) of Eu $\mathrm{In}_{2} A s_{6}$. Horizontal dashed lines in band structure indicate the Fermi level of respective electron concentration $\left(n_{\mathrm{e}}\right)$ and hole concentration $\left(n_{\mathrm{h}}\right)$.

Figure 6(a) shows the thermal conductivity as a function of the temperature. The thermal conductivity of undoped $E_{5} I_{2} \mathrm{As}_{6}$ is 1.6 $\mathrm{W} / \mathrm{mK}$ at $300 \mathrm{~K}$. Furthermore, it decreases monotonically with increasing temperature, likely due to the Umklapp scattering of phonons, and becomes $0.9 \mathrm{~W} / \mathrm{mK}$ at $673 \mathrm{~K}$. All the examined samples show comparable thermal conductivities. However, the $\mathrm{Zn}$ doped samples show a slightly high thermal conductivity because of the increased carrier contribution. The total thermal conductivity consists of the lattice and carrier contributions. The carrier contribution to thermal conductivity is estimated using the Wiedemann-Franz relationship, $\kappa_{\mathrm{el}}=L T \rho^{-1}$, where $L$ is the Lorentz number. We calculated $L$ using the formula $L=1.5+\exp (S / 116)$, where $L$ and $S$ are in units of $10^{-8} \mathrm{~W} \Omega \mathrm{K}^{-2}$ and $\mu \mathrm{V} / \mathrm{K}$, respectively. ${ }^{43}$ We obtained the lattice thermal conductivity by subtracting the electronic component from the total thermal conductivity. As shown in Figure 6(b), the lattice thermal conductivities of these samples are within the experimental uncertainty, which is roughly estimated to be $10 \%$.

It is interesting to note that bipolar contribution to carrier thermal conductivity seems to be negligibly small, unlike 


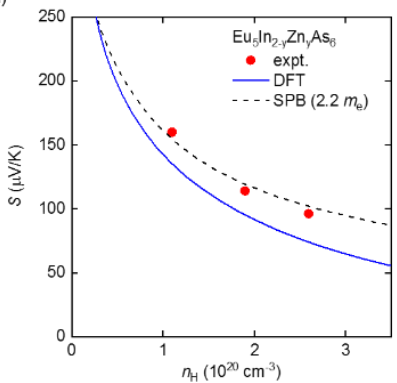

(b)

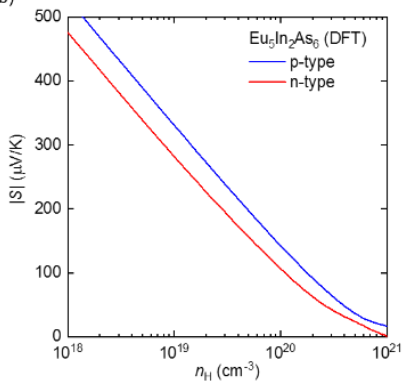

Fig. 9 (a) Hole concentration dependence of the calculated and measured Seebeck coefficients of Eu $\mathrm{In}_{2}$ ${ }_{y} Z_{y} A_{5}$. The calculated data were obtained using the DFT and SPB model with acoustic phonon scattering, assuming an effective mass of $2.2 m_{\mathrm{e}}$, where $m_{\mathrm{e}}$ is the rest mass of a free electron. (b) Carrier concentration dependence of the absolute value of Seebeck coefficients calculated using DFT for $p$-type and $n$-type $\mathrm{Eu}_{5} \mathrm{In}_{2} \mathrm{As}_{6}$.

temperature dependence of Seebeck coefficient shows the contribution from minority carriers. In a two carrier-model, Seebeck coefficient and bipolar thermal conductivity $\left(\kappa_{\mathrm{bip}}\right)$ are expressed as follows, ${ }^{41}$

$$
\begin{gathered}
S=\frac{S_{\mathrm{h}} \sigma_{\mathrm{h}}+S_{\mathrm{e}} \sigma_{\mathrm{e}}}{\sigma_{\mathrm{h}}+\sigma_{\mathrm{e}}} \\
\boldsymbol{\kappa}_{\text {bip }}=\left(S_{\mathrm{h}}-S_{\mathrm{e}}\right)^{2} \frac{\sigma_{\mathrm{h}} \sigma_{\mathrm{e}} T}{\left(\sigma_{\mathrm{h}}+\sigma_{\mathrm{e}}\right)}
\end{gathered}
$$

, where $S_{\mathrm{h}}, S_{\mathrm{e}}, \sigma_{\mathrm{h}}$, and $\sigma_{\mathrm{e}}$ are hole Seebeck coefficient, electron Seebeck coefficient, hole electrical conductivity, and electron electrical conductivity, respectively. Therefore, negligible small bipolar thermal conductivity in $\mathrm{Eu}_{5} \mathrm{In}_{2} \mathrm{As}_{6}$ is most likely due to low $\sigma_{\mathrm{h}}$ and $\sigma_{\mathrm{e}}$.

Furthermore, the lattice thermal conductivity of these samples is comparable to that of the $\mathrm{Sb}$-containing analogue $\mathrm{Eu}_{5} \mathrm{In}_{2} \mathrm{Sb}_{6}{ }^{30}$ This suggests that the low thermal conductivity originates from structural complexity and/or bonding anharmonicity, rather than the heavy constituent elements. For example, a crystal structure characterised by a quasi-one-dimensional chain of InAs $s_{4}$ tetrahedra may contribute to low thermal conductivity. However, a detailed investigation of phonon properties, such as first-principles calculation of phonon dynamics, is required for a further analysis of this phenomenon.

Figure 7 shows the calculated $Z T$ values for $E u_{5-x} \operatorname{La}_{x} \operatorname{In}_{2} A_{6}$ and $E u_{5} I_{2-y} Z n_{y} A s_{6}$. The value of $Z T$ for $E u_{5-x} L_{x} \mid n_{2} A s_{6}$ is less than 0.1, whereas it reaches 0.29 at $773 \mathrm{~K}$ for Eu $\operatorname{In}_{2-y} Z n_{y} A s_{6}$. This is mainly because of the sufficient carrier doping in these samples, as described above.

\section{First-principles calculation}

The first-principles calculations show that $\mathrm{Eu}_{5} \mathrm{In}_{2} \mathrm{As}_{6}$ has a semiconducting band structure, as shown in Figure 8 . The band gap is evaluated to be $0.7 \mathrm{eV}$, which is reasonably consistent with the value experimentally obtained using the temperature dependence of electrical resistivity, $0.58 \mathrm{eV}$. Both the valence band maximum (VBM) and conduction band minimum (CBM) are located on the $\mathrm{Y}-\Gamma$ line. The density of states (DOS) of the VBM has a higher value than that of the CBM. As shown in Figure S3, the VBM is predominantly composed of the As $4 p$ orbital, whereas the CBM is

composed of In $5 s / 5 p$, As $4 p$, and Eu $5 d$ orbitals. We also calculated band structure of $\mathrm{Zn}$-doped $\mathrm{Eu}_{5} \mathrm{In}_{2} \mathrm{As}_{6}$ using virtual crystal approximation. However, the obtained band structure almost coincides with undoped $\mathrm{Eu}_{5} \mathrm{In}_{2} \mathrm{As}_{6}$, except for the Fermi level (results are not shown), indicating that rigid band model can be accepted to investigate the carrier transport of these compounds.

The hole concentration dependence of the calculated and measured Seebeck coefficients is shown in Figure 9(a). Here, we show the results of $E u_{5} I n_{2-y} Z n_{y} A s_{6}$ with $p$-type polarity, because the Hall coefficient was successfully obtained for these samples. The calculated results using DFT are reasonably consistent with the experimental results, although the calculations slightly underestimate the experimental results. We also show the Seebeck coefficient obtained using a single parabolic band (SPB) model with acoustic phonon scattering. ${ }^{44-46}$ The measured Seebeck coefficient is well reproduced with an effective mass of $2.2 \mathrm{me}_{\mathrm{e}}$, where $m_{\mathrm{e}}$ is the rest mass of a free electron. It should be noted that effective mass of undoped $E u_{5} I_{2} A_{6}$ is apparently lower than that of $\mathrm{Zn}$-doped samples. This is because the effective mass analysis using Seebeck coefficient and carrier concentration assumes single parabolic band, while temperature dependence of Seebeck coefficient of undoped $\mathrm{Eu}_{5} \mathrm{In}_{2} \mathrm{As}_{6}$ indicates that this should be regarded as a multicarrier system.

Figure 9(b) shows the Seebeck coefficient obtained using the first-principles calculations for the $p$-type and $n$-type polarity. A higher Seebeck coefficient is obtained for p-type polarity, which is consistent with the higher DOS near the VBM than that near the CBM, as shown in Figure 8.

The calculated temperature dependence of the Seebeck coefficient is shown in Figure S5. For the p-type region, the Seebeck coefficient increases with increasing temperature, which is consistent with the experimental results. On the other hand, the calculated Seebeck coefficient in the $n$-type region also increases almost monotonically with increasing temperature, unlike experiments that show a maximum in the mid-temperature region (Fig. 5). This is likely because of the asymmetry in the relaxation times of electrons and holes, which is assumed to be constant in the theoretical calculation. The asymmetry in the carrier relaxation time is also suggested by the large difference in the weighted mobilities of the p-type and n-type samples, as listed in Table 2.

\section{Discussion}

In the present study, it was shown that $\mathrm{Zn}$ acts as an efficient dopant to induce hole carriers. In contrast, electron doping using La as the dopant appears to be compensated by defects, such as cation ( $\mathrm{Eu}$ and/or In) vacancies. In some Zintl compounds, it has been demonstrated that excess amounts of cations are effective in suppressing cation vacancies, ${ }^{47,48}$ which is conceptualised by the so-called phase boundary mapping. ${ }^{49}$ We preliminarily investigated the synthesis of La-doped $E u_{5} I_{2} A s_{6}$ with excess amounts of Eu or In. Regrettably, these samples show almost comparable thermoelectric transport to those of stoichiometric compounds without excess cations, suggesting that dopant activity is difficult to control via nonstoichiometry. 
We also investigated the Se dopant to increase the electron concentration of Eu $\mathrm{In}_{2} A s_{6}$. However, Se-doped Eu $\mathrm{I}_{5} \mathrm{n}_{2} A s_{6}$ has a significant amount of the $\mathrm{Eu}_{3} \ln _{2} \mathrm{As}_{4}$ impurity phase according to the SXRD measurements. Therefore, we ruled out Se-doped samples for a further investigation of $n$-type $\mathrm{Eu}_{5} \operatorname{In}_{2} \mathrm{As}_{6}$.

\section{Conclusions}

We demonstrated Zintl arsenide $\mathrm{Eu}_{5} \mathrm{In}_{2} \mathrm{As}_{6}$ as a bipolar dopable material. The n-type polarity was confirmed in La-doped $E u_{5} I_{2} A_{6}$, although the electron concentration was apparently lower than the optimal value as indicated from high electrical resistivity. The p-type polarity was observed in the Zn-doped samples, and the hole concentration was optimised, leading to $Z T=0.29$ at $773 \mathrm{~K}$. This doping asymmetry is understood by the difference in the weighted mobility of electrons and holes. The bipolar dopable $\mathrm{Eu}_{5} \mathrm{In}_{2} \mathrm{As}_{6}$ will be a useful platform to facilitate a better understanding of the doping asymmetry in Zintl pnictides.

\section{Conflicts of interest}

There are no conflicts to declare.

\section{Acknowledgements}

This work was partly supported by The Iwatani Naoji Foundation (20-4725), JST CREST (No. JPMJCR16Q6), JSPS KAKENHI (No. 19K15291, 19K15436, 20KK0124), and Tokyo Metropolitan Government Advanced Research (H31-1).

\section{References}

1 G. J. Snyder, E. S. Toberer, Complex thermoelectric materials. Nat. Mater. 2008, 7, 105-114.

2 W. G. Zeier, A. Zevalkink, Z. M. Gibbs, G. Hautier, M. G. Kanatzidis, G. J. Snyder, Thinking like a chemist: intuition in thermoelectric materials. Angew. Chem. Int. Ed. 2016, 55, 6826-6841.

3 L. D. Zhao, V. P. Dravid, M. G. Kanatzidis, The panoscopic approach to high performance thermoelectrics. Energy Environ. Sci. 2014, 7, 251-268.

4 J. R. Sootsman, D. Y. Chung, M. G. Kanatzidis, New and old concepts in thermoelectric materials. Angew. Chem. Int. Ed. 2009, 48, 8616-8639.

5 S. M. Kauzlarich, S. R. Brown, G. J. Snyder, Zintl phases for thermoelectric devices. Dalton Trans. 2007, 21, 2099-2107.

6 E. S. Toberer, A. F. May and G. J. Snyder, Zintl chemistry for designing high efficiency thermoelectric materials, Chem. Mater., 2010, 22, 624-634.

7 J. Shuai, J. Mao, S. Song, Q. Zhang, G. Chen and Z. Ren, Recent progress and future challenges on thermoelectric Zint materials, Mater. Today Phys., 2017, 1, 74-95.
8 X.-J.Wang, M.-B. Tang, H.-H. Chen, X.-X. Yang, J.-T. Zhao, U. Burkhardt, Y. Grin, Synthesis and high thermoelectric efficiency of Zintl phase $\mathrm{YbCd}_{2-x} \mathrm{Zn}_{x} \mathrm{Sb}_{2}$. Appl. Phys. Lett. 2009, 94, 092106

9 J. H. Grebenkemper, Y. Hu, D. Barrett, P. Gogna, C. K. Huang, S. K. Bux and S. M. Kauzlarich, High Temperature Thermoelectric Properties of $\mathrm{Yb}_{14} \mathrm{MnSb}_{11}$ Prepared from Reaction of MnSb with the Elements, Chem. Mater., 2015, 27, 5791-5798.

10 A. Zevalkink, W. G. Zeier, G. Pomrehn, E. Schechtel, W. Tremelb and and G. J. Snyder, Thermoelectric properties of $\mathrm{Sr}_{3} \mathrm{GaSb}_{3}$ - a chain-forming Zintl compound, Energy Environ. Sci., 2012, 5, 9121-9128.

11 A. Zevalkink, J. Swallow, S. Ohno, U. Aydemir, S. Bux and G. J. Snyder, Thermoelectric properties of the $\mathrm{Ca}_{5} \mathrm{Al}_{2-x} \ln _{x} \mathrm{Sb}_{6}$ solid solution, Dalt. Trans., 2014, 43, 15872-15878.

12 S. Ohno, U. Aydemir, M. Amsler, J. H. Pöhls, S. Chanakian, A. Zevalkink, M. A. White, S. K. Bux, C. Wolverton and G. J. Snyder Achieving $\mathrm{zT}>1$ in inexpensive zintl phase $\mathrm{Ca}_{9} \mathrm{Zn}_{4+\mathrm{x}} \mathrm{Sb}_{9}$ by phase boundary mapping, Adv. Funct. Mater., 2017, 27, 1606361-1606370.

13 H. Tamaki, H. K. Sato and T. Kanno, Isotropic conduction network and defect chemistry in $\mathrm{Mg}_{3+\delta} \mathrm{Sb}_{2}$-Based layered zintl compounds with high thermoelectric performance, Adv. Mater., 2016, 28, 10182-10187.

14 J. Zhang, L. Song, S. H. Pedersen, H. Yin, L. T. Hung and B. B. Iversen, Discovery of high-performance low-cost n-type $\mathrm{Mg}_{3} \mathrm{Sb}_{2}$-based thermoelectric materials with multi-valley conduction bands, Nat. Commun., 2017, 8, 13901-13908.

15 C. Chen, W. Xue, S. Li, Z. Zhang, X. Li, X. Wang, Y. Liu, J. Sui, X. Liua, F. Caod, Z. Renf, C.-W. Chuf, Y. Wangc and Q. Zhang, Zintl-phase $\mathrm{Eu}_{2} \mathrm{ZnSb}_{2}$ : a promising thermoelectric material with ultralow thermal conductivity, Proc. Natl. Acad. Sci., 2019, 116, 2831-2836.

16 M. Li, Q. Sun, S. D. Xu, M. Hong, W. Y. Lyu, J. X. Liu, Y. Wang, M. Dargusch, J. Zou and Z. G. Chen, Optimizing Electronic Quality Factor toward High-Performance Ge1-x-yTaxSbyTe Thermoelectrics: The Role of Transition Metal Doping, Adv. Mater., 2021, 33, 1-8.

17 M. Hong, W. Lyv, M. Li, S. Xu, Q. Sun, J. Zou and Z. G. Chen, Rashba Effect Maximizes Thermoelectric Performance of GeTe Derivatives, Joule, 2020, 4, 2030-2043.

18 Y. X. Chen, X. L. Shi, Z. H. Zheng, F. Li, W. Di Liu, W. Y. Chen, X. R. Li, G. X. Liang, J. T. Luo, P. Fan and Z. G. Chen, Twodimensional WSe2/SnSe $p-n$ junctions secure ultrahigh thermoelectric performance in n-type $\mathrm{Pb} / \mathrm{l}$ Co-doped polycrystalline SnSe, Mater. Today Phys., 2021, 16, $100306-$ 100316.

19 Z. Chen, X. Guo, F. Zhang, Q. Shi, M. Tang and R. Ang, Routes for advancing SnTe thermoelectrics, J. Mater. Chem. A, 2020, 8, 16790-16813.

20 S. Xu, X. L. Shi, M. Dargusch, C. Di, J. Zou and Z. G. Chen, Conducting polymer-based flexible thermoelectric materials and devices: From mechanisms to applications, Prog. Mater. Sci., 2021, 121, 100840. 
21 X. L. Shi, H. Wu, Q. Liu, W. Zhou, S. Lu, Z. Shao, M. Dargusch and Z. G. Chen, SrTiO3-based thermoelectrics: Progress and challenges, Nano Energy, 2020, 78, 105195.

22 P. Gorai, A. Goyal, E. S. Toberer and V. Stevanović, A simple chemical guide for finding novel n-type dopable Zintl pnictide thermoelectric materials, J. Mater. Chem. A, 2019, 7, 1938519395.

23 B. R. Ortiz, P. Gorai, T. Braden, E. A. Bensen, S. D. Wilson, V. Stevanović and E. S. Toberer, Discovery of n-Type Zintl phases $\mathrm{RbAlSb}_{4}, \mathrm{RbGaSb}_{4}, \mathrm{CsAlSb}_{4}$, and $\mathrm{CsGaSb}_{4}$, ACS Appl. Energy Mater., 2020, 3, 2182-2191.

24 B. R. Ortiz, P. Gorai, V. Stevanović and E. S. Toberer, Thermoelectric performance and defect chemistry in n-Type Zintl KGaSb 4 , Chem. Mater., 2017, 29, 4523-4534.

25 J. Lin, W. Lv, Y. Gu, K. Guo, X. Yang and J. Zhao, Intentional carrier doping to realize $n$-type conduction in Zintl phases $\mathrm{Eu}_{5-}$ y Lay $_{y} \mathrm{In}_{2.2} \mathrm{Sb}_{6}$, Materials, 2019, 12, 264.

26 K. Rajput, S. Baranets and S. Bobev, Observation of an unexpected n-type semiconducting behavior in the new ternary Zintl phase $\mathrm{Eu}_{3} \ln \mathrm{As}_{3}$, Chem. Mater., 2020, 32, 96169626.

27 A. Balvanz, S. Baranets and S. Bobev, Synthesis and structural characterization of the new Zintl phases $\mathrm{Ba}_{3} \mathrm{Cd}_{2} \mathrm{P}_{4}$ and $\mathrm{Ba}_{2} \mathrm{Cd}_{2} \mathrm{P}_{3}$. Rare example of small gap semiconducting behavior with negative thermopower within the range $300 \mathrm{~K}-700 \mathrm{~K}$, J. Solid State Chem., 2020, 289, 121476.

28 A. B. Childs, S. Baranets and S. Bobev, Five new ternary indium-arsenides discovered. Synthesis and structural characterization of the Zintl phases $\mathrm{Sr}_{3}\left|\mathrm{n}_{2} A s_{4}, B a_{3}\right| \mathrm{n}_{2} A s_{4}$, $\mathrm{Eu}_{3} \mathrm{In}_{2} \mathrm{As}_{4}, \mathrm{Sr}_{5} \mathrm{In}_{2} \mathrm{As} \mathrm{s}_{6}$ and $\mathrm{Eu}_{5} \ln _{2} \mathrm{As} \mathrm{s}_{6}$, J. Solid State Chem., 2019, 278, 120889 .

29 M. Radzieowski, F. Stegemann, S. Klenner, Y. Zhang, B. P. T. Fokwa and O. Janka, On the divalent character of the Eu atoms in the ternary Zintl phases $\mathrm{Eu}_{5} \mathrm{In}_{2} \mathrm{Pn}_{6}$ and $\mathrm{Eu}_{3} \mathrm{MAs}_{3}(\mathrm{Pn}=\mathrm{As}-\mathrm{Bi}$; $\mathrm{M}=\mathrm{Al}, \mathrm{Ga})$, Mater. Chem. Front., 2020, 4, 1231-1248.

30 S. Chanakian, U. Aydemir, A. Zevalkink, Z. M. Gibbs, J. P. Fleurial, S. Bux and G. Jeffrey Snyder, High temperature thermoelectric properties of $\mathrm{Zn}$-doped $\mathrm{Eu}_{5} \mathrm{In}_{2} \mathrm{Sb}_{6}, J$. Mater. Chem. C, 2015, 3, 10518-10524.

31 K. Shinozaki, Y. Goto, K. Hoshi, R. Kiyama, N. Nakamura, A. Miura, C. Moriyoshi, Y. Kuroiwa, H. Usui and Y. Mizuguchi, Thermoelectric Properties of the As/P-Based Zintl Compounds Euln ${ }_{2} \mathrm{As}_{2}(\mathrm{x}=0-2)$ and $\mathrm{SrSn}_{2} \mathrm{As}_{2}$, ACS Appl. Energy Mater. 2021, 4, 5155-5164.

32 Y. Goto, S. Nakanishi, Y. Nakai, T. Mito, A. Miura, C. Moriyoshi, Y. Kuroiwa, H. Usui, T. D. Matsuda, Y. Aoki, Y. Nakacho, Y. Yamada, K. Kanamura and Y. Mizuguchi, The crystal structure and electrical/thermal transport properties of $\mathrm{Li}_{1-x} \mathrm{Sn}_{2+x} \mathrm{P}_{2}$ and its performance as a Li-ion battery anode material, J. Mater. Chem. A, 2021, 9, 7034-7041.

33 S. Kawaguchi, M. Takemoto, K. Osaka, E. Nishibori, C. Moriyoshi, Y. Kubota, Y. Kuroiwa and K. Sugimoto, Highthroughput powder diffraction measurement system consisting of multiple MYTHEN detectors at beamline BL02B2 of SPring-8, Rev. Sci. Instrum., 2017, 88, 085111-085119.
34 V. Petricek, M. Dusek, L. Palatinus, Crystallographic computing system JANA2006: general features. Z. Kristallogr. 2014, 229, 345-352.

35 K. Momma, F. Izumi, VESTA 3 for three-dimensional visualization of crystal, volumetric and morphology data. J. Appl. Crystallogr. 2011, 44, 1272-1276.

36 G. Kresse, J. Furthmüller, Efficient iterative schemes for ab initio total-energy calculations using a plane-wave basis set. Phys. Rev. B 1996, 54, 11169-11186.

37 G. Kresse, D. Joubert, From ultrasoft pseudopotentials to the projector augmented-wave method. D. Phys. Rev. B 1999, 59, 1758-1775.

38 J. P. Perdew, K. Burke, M. Ernzerhof, Generalized gradient approximation made simple. Phys. Rev. Lett. 1996, 77, 38653868.

39 G. K. H. Madsen, and D. J. Singh, BoltzTraP. A code for calculating band-structure dependent quantities. Comput. Phys. Commun. 2006, 175, 67-71.

40 I. D. Brown and R. D. Shannon, Empirical bond-strength-bondlength curves for oxides, Acta Crystallogr. Sect. A, 1973, 29, 266-282.

41 A. F. May, G. J. Snyder, Introduction to modeling thermoelectric transport at high temperatures, Chapter 11 in thermoelectrics and its energy harvesting Vol 1, edited by $D$. M. Rowe. CRC Press (2012).

42 G. J. Snyder, A. H. Snyder, M. Wood, R. Gurunathan, H. Snyder and C. Niu, Weighted mobility, Adv. Mater., 2020, 32, 2001537-2001541.

43 H. S. Kim, Z. M. Gibbs, Y. Tang, H. Wang and G. J. Snyder, Characterization of Lorenz number with Seebeck coefficient measurement, APL Mater., 2015, 3, 041506.

44 A. F. May, E. S. Toberer, A. Saramat and G. J. Snyder, Characterization and analysis of thermoelectric transport in $\mathrm{n}$ type $\mathrm{Ba}_{8} \mathrm{Ga}_{16-\mathrm{x}} \mathrm{Ge}_{30+x}$, Phys. Rev. B, 2009, 80, 125205.

45 L.-D. Zhao, S.-H. Lo, J. He, H. Li, K. Biswas, J. Androulakis, C.-I. Wu, T. P. Hogan, D.-Y. Chung, V. P. Dravid and M. G. Kanatzidis, High performance thermoelectrics from earth-abundant materials: enhanced figure of merit in $\mathrm{PbS}$ by second phase nanostructures., J. Am. Chem. Soc., 2011, 133, 20476-20487.

46 Y. Goto, Y. Sakai, Y. Kamihara and M. Matoba, Effect of Snsubstitution on thermoelectric properties of copper-based sulfide, famatinite $\mathrm{Cu}_{3} \mathrm{SbS}_{4}$, J. Phys. Soc. Jpn., 2017, 84, 044706-044710.

47 W. Zhang, C. Chen, H. Yao, W. Xue, S. Li, F. Bai, Y. Huang, X. Li, X. Lin, F. Cao, J. Sui, S. Wang, B. Yu, Y. Wang, X. Liu and Q. Zhang, Promising Zintl-phase thermoelectric compound SrAgSb, Chem. Mater., 2020, 32, 6983-6989.

48 A. Zevalkink, W. G. Zeier, E. Cheng, J. Snyder, J.-P. Fleurial and S. Bux, Nonstoichiometry in the Zintl phase $\mathrm{Yb}_{1-\delta} \mathrm{Zn}_{2} \mathrm{Sb}_{2}$ as a route to thermoelectric optimization, Chem. Mater., 2014, 26, 5710-5717.

49 S. Ohno, K. Imasato, S. Anand, H. Tamaki, S. D. Kang, P. Gorai, H. K. Sato, E. S. Toberer, T. Kanno and G. J. Snyder, Phase boundary mapping to obtain n-type $\mathrm{Mg}_{3} \mathrm{Sb}_{2}$-based thermoelectrics, Joule, 2018, 2, 1-14. 
Electronic Supplementary Information for

Bipolar doping and thermoelectric properties of Zintl arsenide $\mathrm{Eu}_{5} \mathrm{In}_{2} \mathrm{As}_{6}$

Naoki Tomitaka, ${ }^{\text {a }}$ Yosuke Goto, ${ }^{* a}$ Kota Morino, ${ }^{\text {a }}$ Kazuhisa Hoshi, ${ }^{\text {a }}$ Yuki Nakahira, ${ }^{\text {a }}$ Hiroaki Ito, ${ }^{\mathrm{b}}$ Akira Miura, ${ }^{\mathrm{b}}$ Hidetomo Usui, ${ }^{{ }^{\mathrm{c}}}$ Yoshikazu Mizuguchi ${ }^{\mathrm{a}}$

${ }^{a}$ Department of Physics, Tokyo Metropolitan University, 1-1 Minami-osawa, Hachioji, Tokyo 192-0397, Japan

${ }^{b}$ Faculty of Engineering, Hokkaido University, Kita 13, Nishi 8 Sapporo 060-8628, Japan ${ }^{c}$ Department of Physics and Materials Science, Shimane University, Matsue, 690-8504, Japan

e-mail:y_goto@tmu.ac.jp,hidetomo.usui@riko.shimane-u.ac.jp 

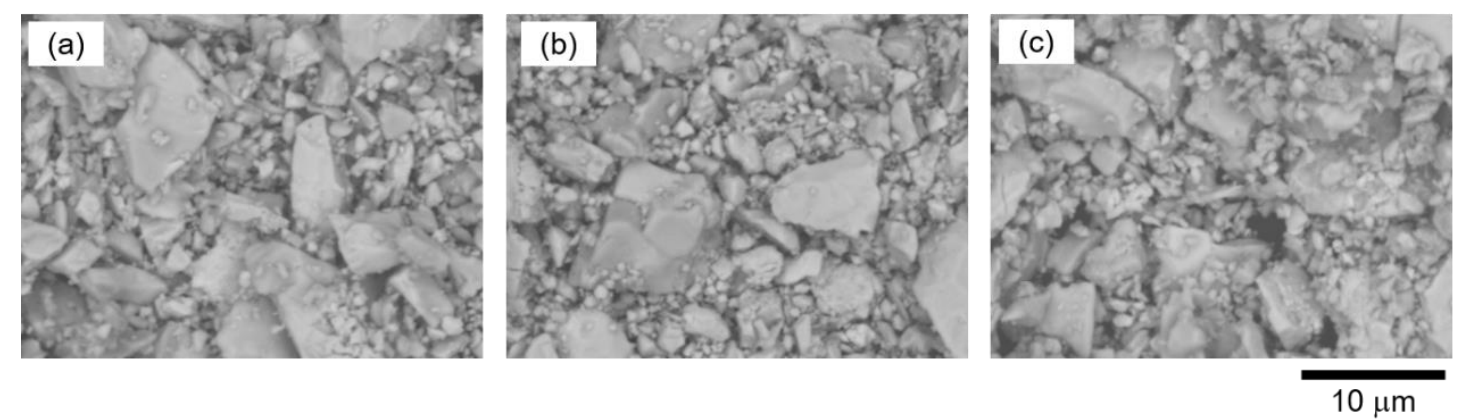

Figure S1. SEM images of pulverized powder of (a) $\mathrm{Eu}_{5} \mathrm{In}_{2} \mathrm{As}_{6}$, (b) $\mathrm{Eu}_{4.90} \mathrm{La}_{0.10} \mathrm{In}_{2} \mathrm{As}_{6}$, and (c) $\mathrm{Eu}_{5} \mathrm{In}_{1.90} \mathrm{Zn}_{0.10} \mathrm{As}_{6}$.
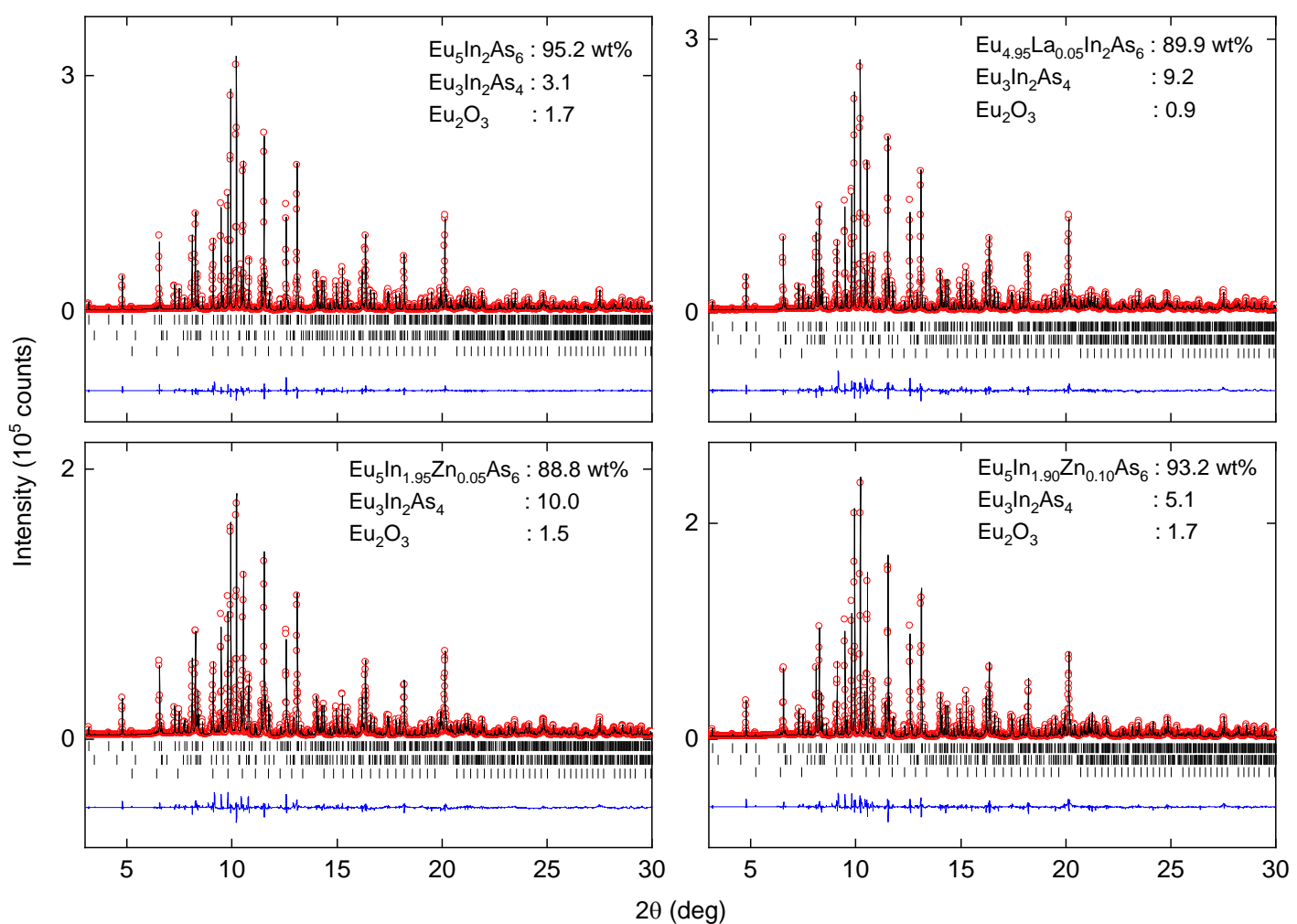

Figure S2

Observed synchrotron X-ray diffraction pattern and the Rietveld refinement results. The circles (red) and solid lines (black) represent the observed and calculated patterns, respectively. The difference between the observed and calculated patterns is shown at the bottom (blue). The vertical marks indicate the Bragg reflection positions for $\mathrm{Eu}_{5} \mathrm{In}_{2} \mathrm{As}_{6}{ }^{-}$ type phase, $\mathrm{Eu}_{3} \mathrm{In}_{2} \mathrm{As}_{4}$, and $\mathrm{Eu}_{2} \mathrm{O}_{3}$, respectively, from top to bottom. Amount of these phases are denoted in the inset. 
Table S1

Reliability factors of the Rietveld refinement of $\mathrm{Eu}_{5-x} \operatorname{La}_{x} \operatorname{In}_{2} \mathrm{As}_{6}$ and $\mathrm{Eu}_{5} \operatorname{In}_{2-y} \mathrm{Zn}_{y} \mathrm{As} 6$.

\begin{tabular}{cccc}
\hline Sample & $R_{\mathrm{wp}}(\%)$ & $R_{\mathrm{p}}(\%)$ & $\mathrm{GOF}$ \\
\hline $\mathrm{Eu}_{5-x} \mathrm{La}_{x} \operatorname{In}_{2} \mathrm{As}_{6}$ & & & \\
$x=0$ & 5.37 & 5.17 & 7.17 \\
0.05 & 8.70 & 7.92 & 11.17 \\
0.10 & 8.35 & 6.78 & 9.70 \\
$\mathrm{Eu}_{5} \mathrm{In}_{2-y} \mathrm{Zn}_{y} \mathrm{As}_{6}$ & & & \\
$y=0.05$ & 5.58 & 5.77 & 7.93 \\
0.10 & 6.94 & 6.97 & 9.80 \\
\hline
\end{tabular}

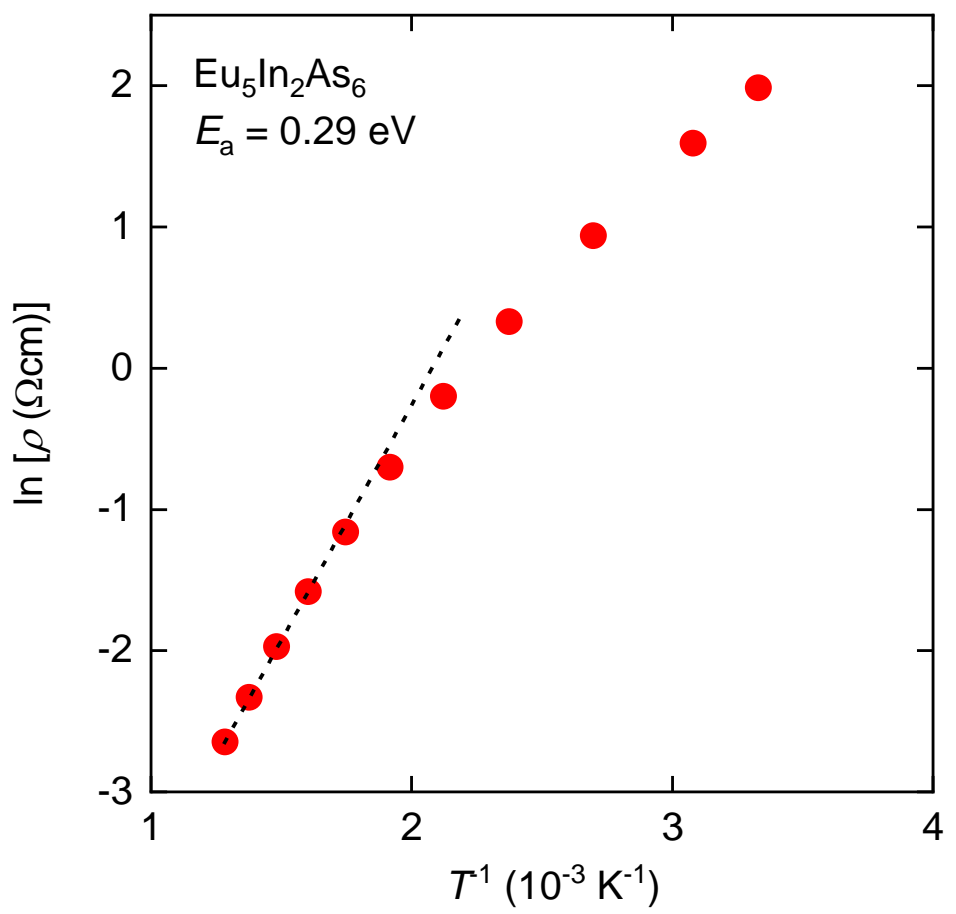

Figure S3

Temperature $(T)$ dependence of electrical resistivity $(\rho)$ of $\mathrm{Eu}_{5} \mathrm{In}_{2} \mathrm{As} 6$. Activation energy $\left(E_{\mathrm{a}}\right)$ was obtained using measurement results from 623 to $778 \mathrm{~K}$. 


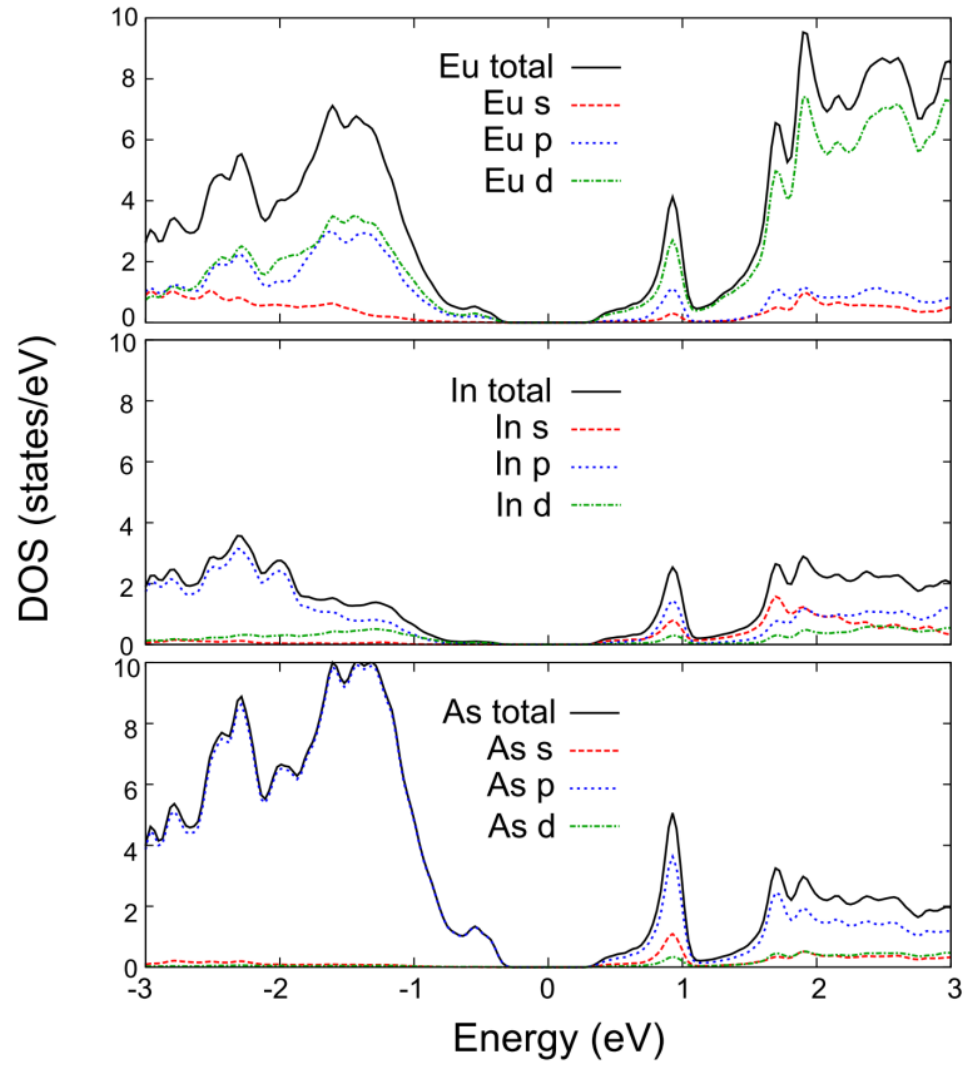

Figure S4

Partial density of states (DOS) of $\mathrm{Eu}_{5} \operatorname{In}_{2} \mathrm{As}_{6}$. 


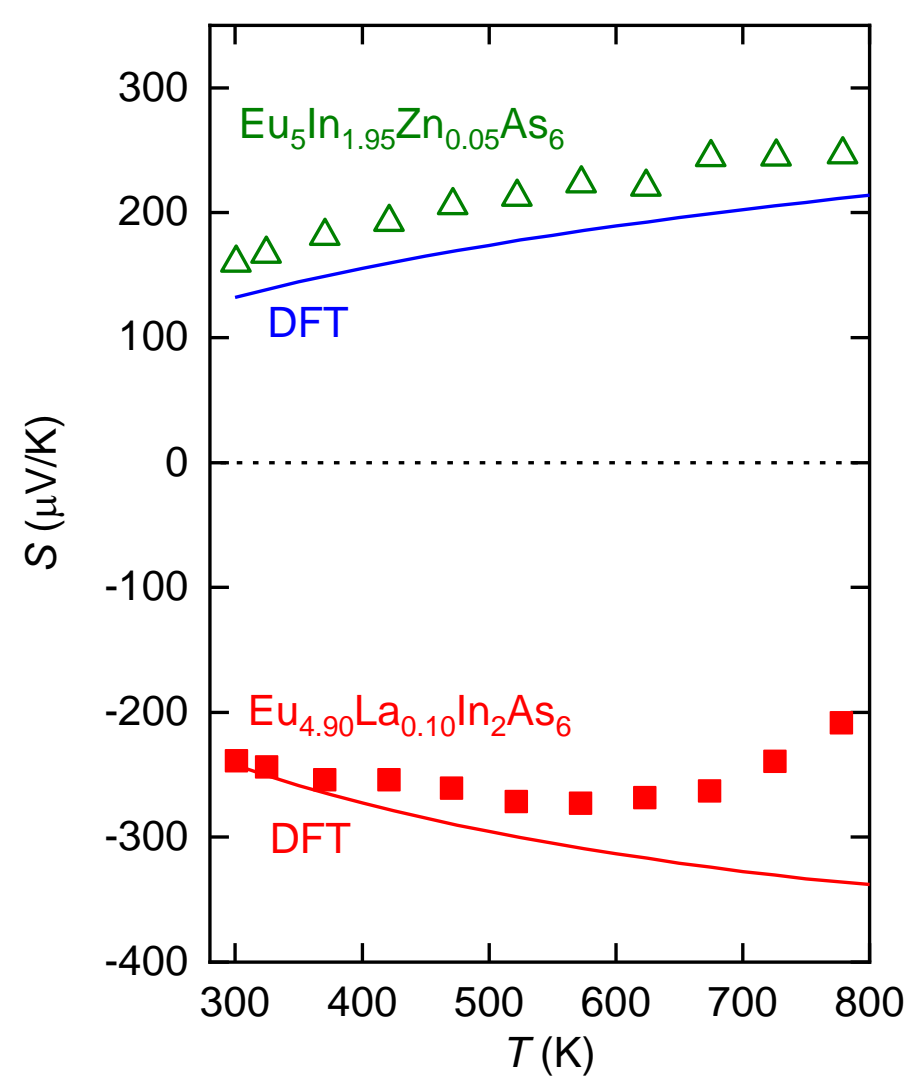

Figure S5

Temperature $(T)$ dependence of Seebeck coefficient $(S)$ of $\mathrm{Eu}_{5} \operatorname{In}_{1.95} \mathrm{Zn}_{0.05} \mathrm{As}_{6}$ and $\mathrm{Eu}_{4.90} \mathrm{La}_{0.10} \mathrm{In}_{2} \mathrm{As}_{6}$. Calculated $S$ using density functional theory (DFT) is also shown. Measured hole concentration $\left(1.1 \times 10^{20} \mathrm{~cm}^{-3}\right)$ was used to plot calculated results for $\mathrm{p}$ type region, while electron concentration of $1.6 \times 10^{19} \mathrm{~cm}^{-3}$ was assumed for n-type region, because reliable Hall coefficient cannot be obtained for n-type La-doped samples. 


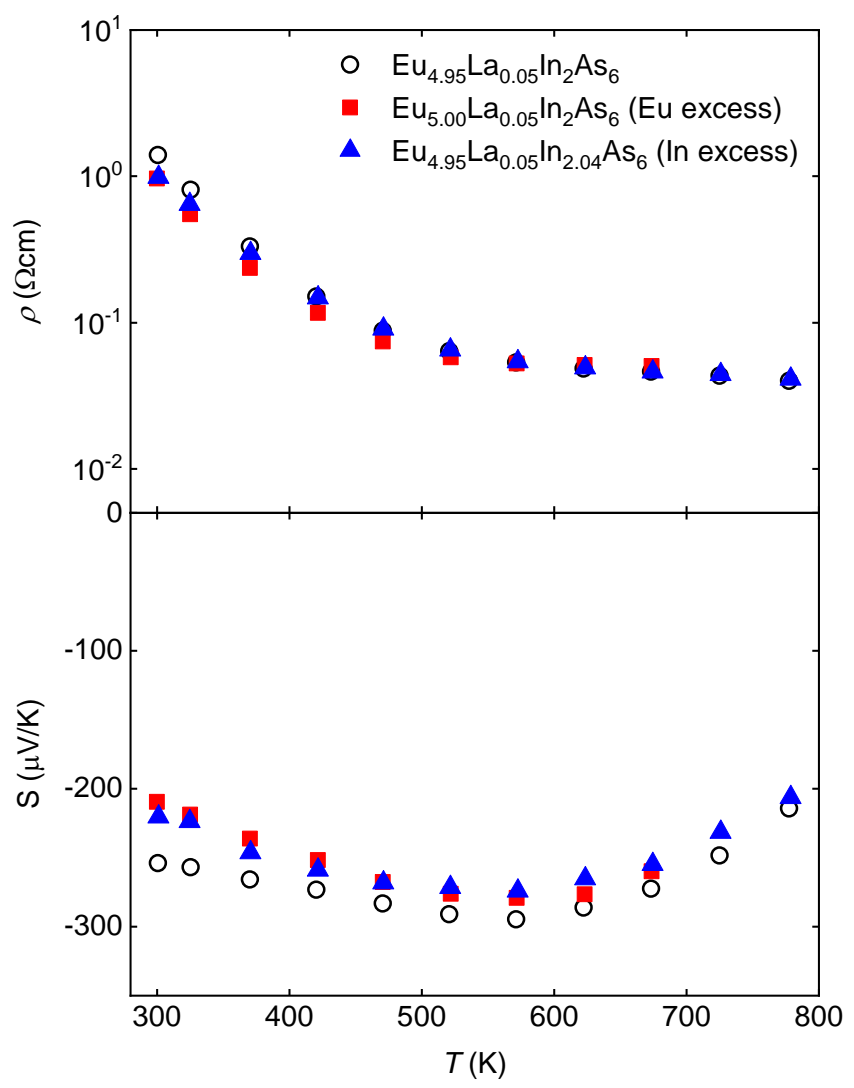

Figure S6

Temperature $(T)$ dependence of electrical resistivity $(\rho)$ and Seebeck coefficient $(S)$ of La-doped $\mathrm{Eu}_{5} \mathrm{In}_{2} \mathrm{As} 6$ synthesized with excess amount of Eu or La. Nominal composition of starting materials is denoted in the inset. 